\title{
A polymorphic minisatellite region of BORIS regulates gene expression and its rare variants correlate with lung cancer susceptibility
}

\author{
Se-Lyun Yoon ${ }^{1,7}$, Yun-Gil Roh ${ }^{1,7}$, In-Sun $\mathrm{Chu}^{2}$, Jeonghoon $\mathrm{Heo}^{3}$, Seung Il Kim ${ }^{4}$, Heekyung Chang ${ }^{5}$, \\ Tae-Hong Kang ${ }^{1}$, Jin Woong Chung ${ }^{1}$, Sang Seok Koh ${ }^{1}$, Vladimir Larionov ${ }^{6}$ and Sun-Hee Leem ${ }^{1}$
}

\begin{abstract}
Aberrant expression of BORIS/CTCFL (Brother of the Regulator of Imprinted Sites/CTCF-like protein) is reported in different malignancies. In this study, we characterized the entire promoter region of BORIS/CTCFL, including the CpG islands, to assess the relationship between BORIS expression and lung cancer. To simplify the construction of luciferase reporter cassettes with various-sized portions of the upstream region, genomic copies of BORIS were isolated using TAR cloning technology. We analyzed three promoter blocks: the GATA/CCAAT box, the CpG islands and the minisatellite region BORIS-MS2. Polymorphic minisatellite sequences were isolated from genomic DNA prepared from the blood of controls and cases. Of the three promoter blocks, the GATA/CCAAT box was determined to be a critical element of the core promoter, while the $\mathrm{CpG}$ islands and the BORIS-MS2 minisatellite region were found to act as regulators. Interestingly, the polymorphic minisatellite region BORIS-MS2 was identified as a negative regulator that repressed the expression levels of luciferase reporter cassettes less effectively in cancer cells compared with normal cells. We also examined the association between the size of BORIS-MS2 and lung cancer in a case-control study with 590 controls and 206 lung cancer cases. Rare alleles of BORIS-MS2 were associated with a statistically significantly increased risk of lung cancer (odds ratio, 2.04; 95\% confidence interval, 1.02-4.08; and $P=0.039$ ). To conclude, our data provide information on the organization of the BORIS promoter region and gene regulation in normal and cancer cells. In addition, we propose that specific alleles of the BORIS-MS2 region could be used to identify the risk for lung cancer.
\end{abstract}

Experimental \& Molecular Medicine (2016) 48, e246; doi:10.1038/emm.2016.50; published online 15 July 2016

\section{INTRODUCTION}

The BORIS/CTCFL (Brother of the Regulator of Imprinted Sites/CTCF-like protein) gene is classified as a member of the cancer-testis antigen (CTA) family; it is expressed in the testis during spermatogenesis, ${ }^{1,2}$ as well as in multiple cancers, including uterine (endometrial), breast, lung and gastric cancers. ${ }^{3-6}$ High levels of the BORIS protein in the leukocytes of patients with breast cancer were detected, which suggests that BORIS can be used as a valuable marker. ${ }^{7}$ Moreover, a $\mathrm{CpG}$ island in the promoter region of BORIS is known to be a key factor regulating gene expression, ${ }^{4,8}$ and BORIS expression in human cancer cells has been proposed to lead to epigenetic deregulation. Overexpression of BORIS has been correlated with the hypomethylation of its promoter in prostate and ovarian cancers in several reports, ${ }^{9,10}$ and partial demethylation of the BORIS promoter has been detected in ovarian, ${ }^{11}$ colon $^{11}$ and lung cancer ${ }^{8}$ and in leukemic cell lines. ${ }^{11}$ However, in our previous study, no correlation between methylation status and BORIS gene expression in gastric cancer tissues was detected. ${ }^{6}$

To elucidate the regulation of BORIS during tumorigenesis, in a previous study, we characterized the entire genomic region of the BORIS locus including the promoter region in gastric cancer cells. ${ }^{12}$ A CpG island ( -1096 to -762 from the first ATG) and two minisatellites (variable number of tandem repeats; BORIS-MS1 and BORIS-MS2) were identified through the characterization of the genomic DNA sequence upstream of

\footnotetext{
${ }^{1}$ Department of Biological Science, Dong-A University, Busan, Korea; ${ }^{2}$ Korean Bioinformation Center, Korea Research Institute of Bioscience and Biotechnology, Daejeon, Korea; ${ }^{3}$ Department of Molecular Biology and Immunology, Kosin University College of Medicine, Busan, Korea; ${ }^{4}$ Drug \& Disease Target Team, Korea Basic Science Institute, Daejeon, Korea; ${ }^{5}$ Department of Pathology, Kosin University College of Medicine, Busan, Korea and ${ }^{6}$ Developmental Therapeutic Branch, National Cancer Institute, Bethesda, MD, USA

${ }^{7}$ These authors contributed equally to this work.

Correspondence: Professor S-H Leem, Department of Biological Science, Dong-A University, 37 Nakdong-Daero 550 Beon-gil, Saha-gu, Busan 49315 , Korea.
}

E-mail: shleem@dau.ac.kr

Received 18 December 2015; revised 20 January 2016; accepted 21 January 2016 
the gene-coding region. ${ }^{12}$ In this study, we assessed the expression and methylation status of the BORIS gene in lung cancer tissues and re-analyzed the regulatory elements, that is, the CpG island and two minisatellites, in its promoter region to determine whether these elements are necessary for expression of a reporter gene in lung cancer cells. In addition, we described two well-positioned elements that contained the binding sequences for GATA- $1^{13}$ and the CCAAT box. ${ }^{14}$

Lung cancer is the most commonly diagnosed cancer and the leading cause of death in males. In addition, it is the fourth most common cancer in females worldwide, based on GLOBOCAN 2008 estimates, ${ }^{15}$ and it is a major public health problem in Korea. Because some minisatellite alleles are associated with human diseases, ${ }^{16,17}$ we investigated the relationship between cancer predisposition and minisatellite variants in BORIS $S^{6,12}$ and revealed increased susceptibility in young patients with breast cancer containing short rare minisatellite alleles of BORIS-MS2. To determine whether allelic variation in BORIS minisatellites influences susceptibility to lung cancer, a case-control study was performed using a PCR-based method. Here, we report that rare allelic variants of BORIS-MS2 are correlated with lung cancer susceptibility in a Korean population. In addition, to assess whether this minisatellite region has a role in gene regulation, the transcriptional levels of a reporter gene linked to the minisatellites and driven by the BORIS promoter in lung cell lines were examined.

\section{MATERIALS AND METHODS}

\section{Study population and genotyping assays for BORIS-MS2}

To examine the minisatellite polymorphisms in BORIS-MS2, a casecontrol study was performed with 590 cancer-free controls and 206 lung cancer cases. The controls and cases had similar characteristics regarding sex and age (control average age, 60.5 years, range 30-83 years; patient average age, 63.0 years, range $32-82$ years) (Table 1 ). Controls were selected from the Department of Preventive Medicine and Internal Medicine at Dong-A University hospitals between 1997 and 2004 (Busan, Korea). ${ }^{12}$ The control group, with no personal history of cancer or current cancer, was recruited and completed an interview. The biospecimens from lung cancer patients in this study were provided by the Chungbuk National University Hospital, a member of the National Biobank of Korea, which is supported by the Ministry of Health, Welfare and Family Affairs and Dong-A University Hospital in Busan, Korea. All samples from the National Biobank of Korea were obtained with informed consent and institutional review board-approved protocols. The Committee of Bioethics of Dong-A University approved the procedure and design of this study (\#IRB-06-10-02 \& IRB-07-10-7; Busan, Korea).

For genotyping, genomic DNA was isolated from peripheral leukocytes, which were isolated from $400 \mu \mathrm{l}$ of whole blood using a Blood and Cell Culture DNA Mini Kit (Qiagen, Valencia, CA, USA). The genotyping assay of the minisatellite polymorphism was described previously ${ }^{12}$ with the PCR primer pair $5^{\prime}$-CTTGGGAGACCTGGGGG ATGAATAG-3' (forward) and 5'-GCACCCCATTCCCCATCCTC-3' (reverse) for BORIS-MS2. The PCR products were separated on a $1.2 \%$ agarose gel at $80 \mathrm{~V}$ for $4 \mathrm{~h}$ and then stained with ethidium bromide.

\section{RNA isolation and reverse transcription}

Total RNA was isolated from tissues using TRIzol reagent (Invitrogen, Carlsbad, CA, USA) according to the manufacturer's instructions. The Qiagen RNeasy Mini kit (Qiagen) was used for isolation of total RNA from cell lines and lung cancer tissue specimens. For the reverse transcription reaction, a mixture of total RNA $(3 \mu \mathrm{g})$, an oligo $(\mathrm{dT})_{20}$ $(50 \mathrm{~nm})$ and $10 \mathrm{~mm} \mathrm{dNTP}$ were incubated at $65^{\circ} \mathrm{C}$ for $5 \mathrm{~min}$. A mixture of $1 \times$ RT buffer, $25 \mathrm{~nm} \mathrm{MgCl}_{2}, 0.1 \mathrm{~m}$ DTT and RNaseOUT were incubated at $42{ }^{\circ} \mathrm{C}$ for $2 \mathrm{~min}$. After incubation, Invitrogen

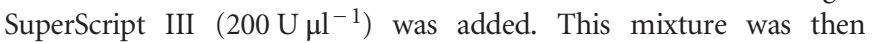
incubated at $42^{\circ} \mathrm{C}$ for $50 \mathrm{~min}$ followed by $70^{\circ} \mathrm{C}$ for $15 \mathrm{~min}$. RNase

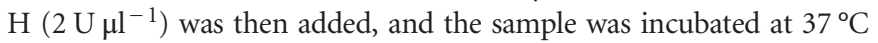
for $20 \mathrm{~min}$ in a 9700 Thermocycler (Perkin-Elmer, Waltham, MA, USA) as described previously. ${ }^{12}$

\section{Bisulfate sequencing of the BORIS promoter regions}

Genomic DNA was subjected to bisulfite modification using the EZ DNA Methylation-Gold Kit (Zymo Research, Irrine, CA, USA). Each sequence was amplified using G-Taq polymerase (Cosmo Genetech, Seoul, Korea) as previously described. ${ }^{6}$ PCR fragments were gel extracted using a Gel Extraction kit (Qiagen) and ligated into the Mighty TA cloning vector (TaKaRa, Kusatsu, Shiga, Japan). Following transformation, plasmids from individual bacterial colonies were isolated and sequenced. Using bisulfate sequencing, the methylation status of the BORIS promoter region was determined and represented

Table 1 Age and sex distribution of cases and controls

\begin{tabular}{|c|c|c|c|c|c|c|}
\hline \multirow[b]{2}{*}{ Characteristic } & \multicolumn{3}{|c|}{ Controls, N (\%) } & \multicolumn{3}{|c|}{ Lung cancer cases, N (\%) } \\
\hline & Males & Females & Total & Males & Females & Total \\
\hline \multicolumn{7}{|l|}{ Age (years) } \\
\hline $30-39$ & $7(2.1)$ & $21(8.0)$ & $28(4.7)$ & $2(1.2)$ & $2(5.7)$ & $4(1.9)$ \\
\hline $40-49$ & $21(6.4)$ & $43(16.3)$ & $64(10.8)$ & $10(5.8)$ & $4(11.4)$ & $14(6.8)$ \\
\hline $50-59$ & $71(21.8)$ & $71(26.9)$ & $142(24.1)$ & 37 (21.6) & $7(20.0)$ & $44(21.4)$ \\
\hline $60-69$ & 153 (46.9) & $81(30.7)$ & $234(39.7)$ & $84(49.1)$ & $11(31.4)$ & $95(46.1)$ \\
\hline 70-79 & 68 (20.9) & 42 (15.9) & 110 (18.6) & 34 (19.9) & $9(25.7)$ & 43 (20.9) \\
\hline$\geqslant 80$ & $6(1.8)$ & $6(2.3)$ & $12(2.0)$ & $4(2.3)$ & $2(5.7)$ & $6(2.9)$ \\
\hline Average & 62.9 & 57.5 & 60.5 & 63.4 & 61.1 & 63.0 \\
\hline Median & 64 & 56 & 62 & 64 & 64 & 64 \\
\hline$N$ & 326 & 264 & 590 & 171 & 35 & 206 \\
\hline
\end{tabular}


a

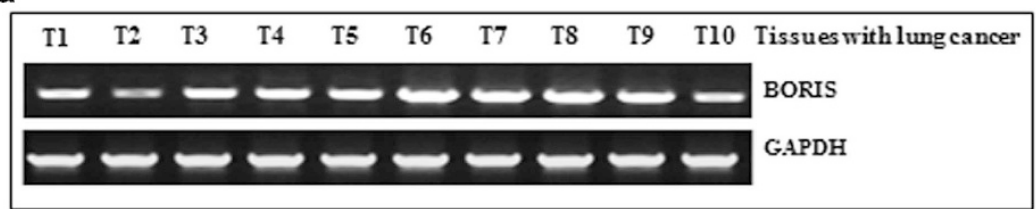

b

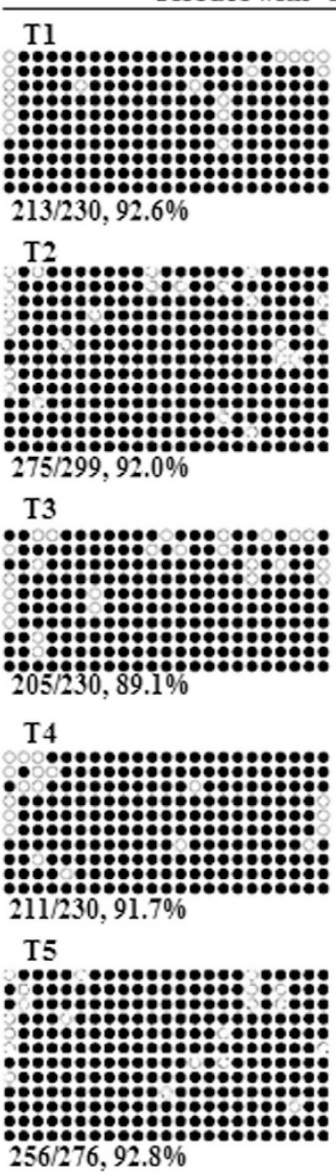

Lung cancer cell line

T6
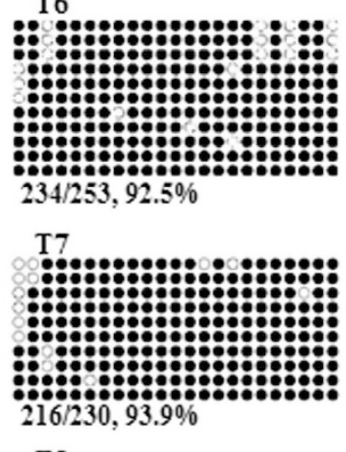

T8

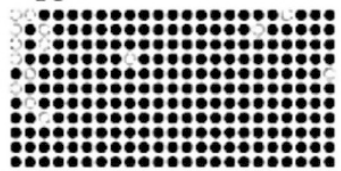

$238 / 253,94.1 \%$

T9

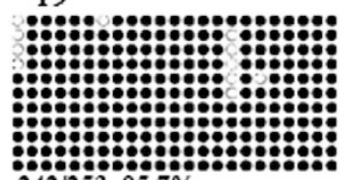

$242 / 253,95.7 \%$

$\mathrm{T} 10$

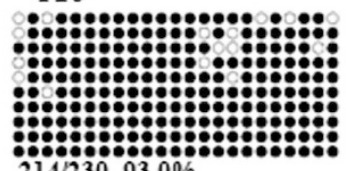

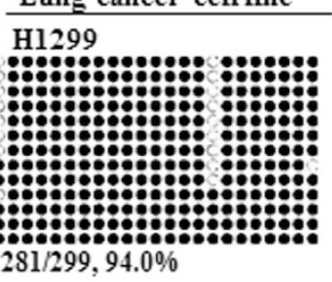

H1299, 5-azad-C $1 \mu \mathrm{M}$

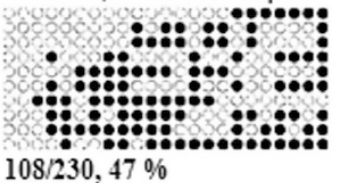

Hl299, 5-azad-C $5 \mu \mathrm{M}$

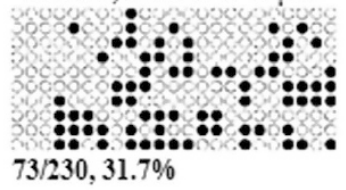

Figure 1 Gene expression and methylation status of the $5^{\prime}$ flanking non-coding region of BORIS. (a) Gene expression of BORIS in tumor tissues (T1-T10) of lung cancer patients. (b) Results of bisulfite sequencing analysis of a $\mathrm{CpG}$ island in the $5^{\prime}$ flanking region of BORIS in tumor tissues (T) and lung cancer cells (H1299 cells and H1299 cells treated with 1 and $5 \mu \mathrm{m}$ 5-azad-C). Open circles (O) indicate non-methylated $\mathrm{CpG}$; filled circles (๑) indicate methylated CpG; percentages indicate the ratio of methylation.

as follows: open circles indicate non-methylated CpG and filled circles indicate methylated CpG (Figure 1).

\section{Plasmid construction}

To generate the luciferase reporter vectors with various fragments of the BORIS $5^{\prime}$-promoter region, fragments were amplified from a bacterial artificial chromosome clone (Supplementary Figure S1) containing the BORIS genomic sequence by PCR and inserted into the KpnI/NheI sites of the luciferase reporter vector pGL3-Basic (Promega, Madison, WI, USA). The primers and the plasmids in this study are listed in Supplementary Table S1. To characterize the effect of BORIS-MS2 on BORIS expression, part of the BORIS promoter p700 ( -1108 to -425$)$ was cloned and introduced into the BglII/ HindIII sites of the luciferase reporter vector pGL3-Basic to generate the p700 fragment (Figure 2). Mutated constructs (GATA-1 $\Delta \# 1$, GATA- $1 \Delta \# 2$ and CCAAT $\Delta$ ) were prepared by substitution of the
GATA-1 site and CCAAT box in the p700 construct as shown in Figure 3. Two common (TR14 and TR15) alleles and five rare alleles (TR10, TR13, TR16, TR17 and TR18) were amplified from genomic DNA derived from controls and cases and inserted into the KpnI/BglII sites of the p700 fragment to generate the reporter plasmids p700 +TR10, p700+TR13, p700+TR14, p700+TR15, p700+TR16, p700 +TR17 and p700+TR18 (Figure 4). All constructs were confirmed by DNA sequencing.

Cells and luciferase assays

The following human cell lines were tested for the effect of BORISMS2 on BORIS expression: 293T/HEK293T (human embryonic kidney cell line obtained from the Korean Cell Line Bank (KCLB, Seoul, Korea), H1299 (lung cancer cell line from the American Type Culture Collection (ATCC, Manassas, VA, USA) and MDA-MB-231 (breast cancer cell line from KCLB). For the luciferase assay, cells 
a

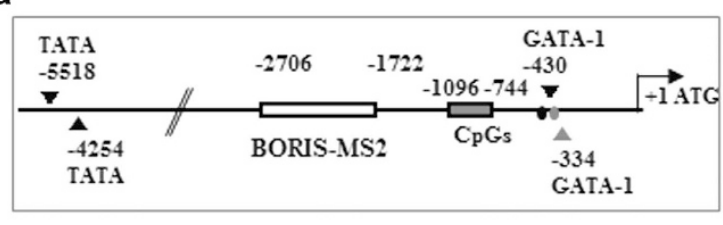

b

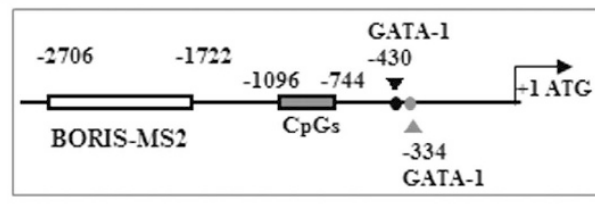

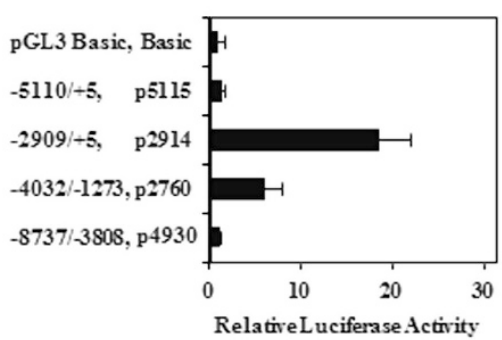

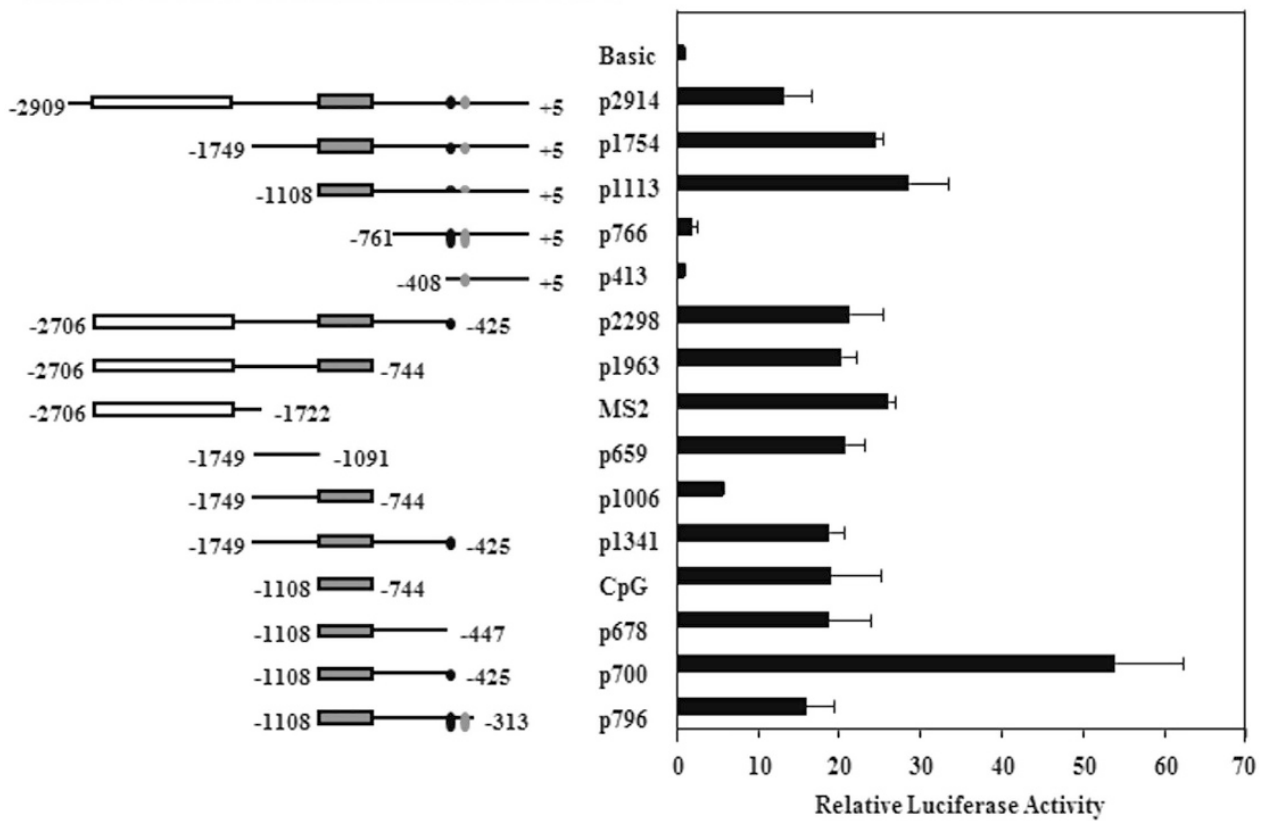

Figure 2 Activity analysis of the BORIS promoter. (a) Structure of the $\sim 6-\mathrm{kb}$ upstream region of BORIS. The figure in the box shows the putative binding sites for TATA and GATA-1, the minisatellite region (BORIS-MS2) and the CpG islands. The position of the CCAAT box is shown in the figure. The results of the luciferase assay with the empty control vector and the BORIS promoter vectors with four different regions are shown on the right. (b) Expression of BORIS promoter-luciferase reporters. The upper boxed panel shows the positions of BORIS-MS2, the CpG islands and the putative binding sites for GATA-1 in the p2914 vector, which represents the highest expression level in the above study. The structures of the BORIS luciferase reporter vectors are shown on the left. The results of the luciferase assay are shown on the right. Firefly luciferase activities of at least four independent experiments were calculated after normalization to Renilla luciferase values.

$\left(1 \times 10^{5}\right)$ were seeded in 12-well plates, cultured overnight and transfected with the BORIS promoter-luciferase plasmids $(0.5 \mu \mathrm{g}$ per well) using the FuGENE6 transfection reagent (Roche Diagnostics, Indianapolis, IN, USA) at a ratio of DNA/FuGENE6 of 1:3. Analysis of the cells was performed using a dual-luciferase reporter assay system (Promega) $48 \mathrm{~h}$ after completion of the transfection procedure. Firefly luciferase activities were normalized to Renilla luciferase activity, and activity was expressed in relative luciferase units to reflect the promoter activity. Triplicate transfections of each construct were tested for each experiment, and the final results were calculated from four independent experiments.

\section{Statistical analysis}

Regression analyses were carried out to determine the odds ratios (ORs) of association between the control and case groups. ORs were predicted using the natural logarithm and its s.e. Where relevant, a $\chi^{2}$-test was performed with one degree of freedom for statistical significance. Differences were regarded as significant with confidence intervals (CIs) of 95\%. All tests were two-sided, with $P<0.05$ considered statistically significant. Statistical analyses were performed using MS Excel with CHITEST and R statistical software (v2.5.1, www.r-project.org) with $\chi^{2}$-test for the $\chi^{2}$-calculation values. The Kaplan-Meier plot was used in the R program version 2.10.0 (https:// www.r-project.org/).

\section{RESULTS}

BORIS expression and methylation status in lung cancer tissues

BORIS gene expression was examined in 20 cell lines, which included normal fibroblast, osteosarcoma, melanoma, breast cancer, prostate cancer, colon cancer, lung cancer and gastric cancer cell lines from a previous study. ${ }^{6}$ Compared with the 
a

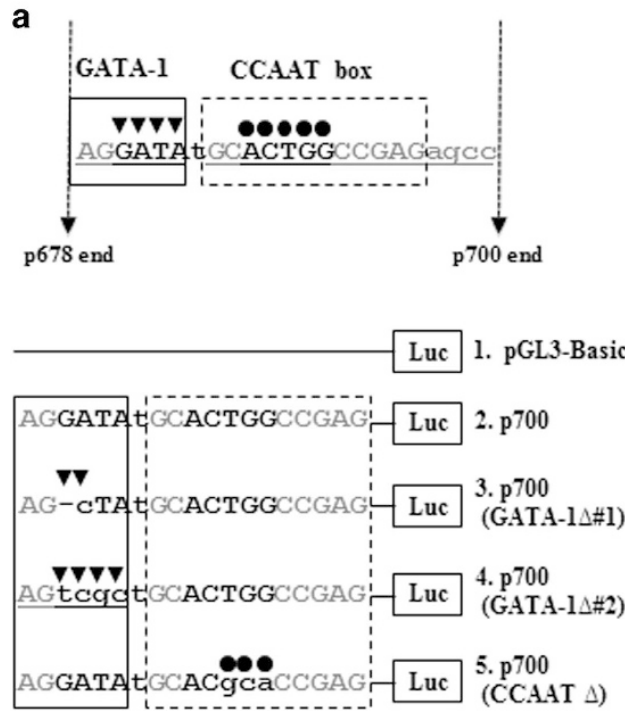

b

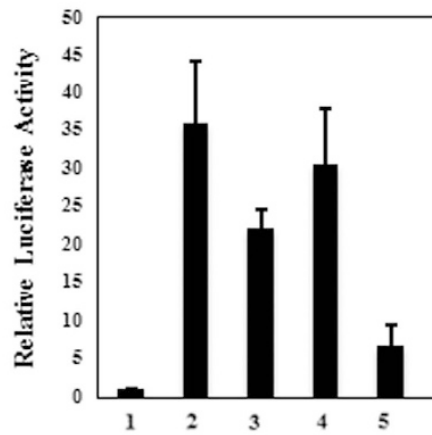

\begin{tabular}{l|c|c|c}
\hline & Vector & Ave. $\pm \mathrm{SE}$ & $\boldsymbol{P}$ \\
\hline $\mathbf{l}$ & pGL3-Basic & $1 \pm 0.05$ & - \\
$\mathbf{2}$ & p700 & $36.06 \pm 8.11$ & $3.38 \mathrm{E}-19$ \\
$\mathbf{3}$ & GATA-1 $\Delta=1$ & $22.34 \pm 2.23$ & $1.58 \mathrm{E}-24$ \\
$\mathbf{4}$ & GATA-1 $\Delta=2$ & $30.73 \pm 7.32$ & $1.40 \mathrm{E}-15$ \\
$\mathbf{5}$ & CCAAT $\Delta$ & $6.80 \pm 2.55$ & $4.49 \mathrm{E}-10$ \\
\hline
\end{tabular}

Figure 3 Analysis of the core promoter of BORIS. (a) The upper panel shows the positions of putative binding sites for GATA-1 and the CCAAT box in the p700 vector, which had the highest expression in Figure $2 \mathrm{~b}$. This region represents the difference between the p678 and p700 vectors. The wild-type construct (p700) contained putative binding sites for GATA-1 and the CCAAT box, whereas the mutant constructs (GATA-1 $\Delta \# 1$, GATA- $1 \Delta \# 2$ and CCAAT $\Delta$ ) were mutated at the GATA-1 site or the CCAAT box in the p700 vector. (b) Luciferase activity of each vector is shown in the upper panel. The lower table shows the relative luciferase activity compared with control vector as the mean \pm s.e. and the $P$-values of at least four independent experiments.

normal cells, various cancer cells, including osteosarcoma, melanoma, breast cancer, prostate cancer, colon cancer, lung cancer and gastric cancer showed abnormal expression of BORIS. Among the lung cancer cell lines (A549, H358, H460, H1299), H1299 cells showed the highest expression. ${ }^{6}$ Then, we investigated BORIS gene expression in lung tumor tissues (Figure 1). In 10 lung tumor tissues, the BORIS levels were different; the lowest levels were observed in 2 cancer tissues (T2 and T10), moderately increased levels were observed in 4 cancer tissues (T1, T3, T4 and T5) and the highest levels were detected in 4 cancer tissues (T6, T7, T8 and T9) at the same level as the H1299 cancer cell line (Figure 1a). To analyze the possible relationship between BORIS gene activity and epigenetic changes in lung cancer, we assessed DNA methylation levels in these tumor tissues. As previously reported in gastric cancer tissues, ${ }^{6}$ methylation $(89.1-95.7 \%)$ of the BORIS promoter region in lung cancer tissues was almost the same despite the different degree of BORIS expression (Figure 1b). The lung cancer cell line H1299 also showed similar levels of methylation (94\%), but cells treated with 5-azad-C (1 and $5 \mu \mathrm{M})$ showed decreased methylation levels (31.7-47\%). We next extensively analyzed the BORIS promoter region to elucidate the regulation of this gene during tumorigenesis.

Cloning of the 5'-regulatory region of the BORIS gene and characterization of transcriptional activities of the promoter We cloned the genomic fragment containing the entire BORIS gene region (Supplementary Figure S1) from human genomic DNA using the TAR cloning method. ${ }^{18}$ Two clones containing $\mathrm{a} \sim 70-\mathrm{kb}$ genomic fragment were isolated and found to carry the entire BORIS gene (Supplementary Figures S1B and C).
The YAC clone was identified by screening a mini-YAC library with PCR using a set of diagnostic primers (Supplementary Table S1). The YAC includes the BORIS-coding region, the $\sim 30 \mathrm{~kb} 5^{\prime}$ upstream region and the $\sim 8 \mathrm{~kb} 3^{\prime}$ flanking regions. TAR cloning of BORIS in yeast greatly simplifies PCR amplification of the promoter regions enriched by repeated and CG-rich sequences, which are poorly amplified from human genomic DNA. We constructed the various reporter vectors (see Materials and Methods, Supplementary Table S1, Figures 2,3,4) using this clone (BORIS I, Supplementary Figures S1B and C),

Transient transfection of 293T cells with the cloned - 2909/ +5 genomic fragment (p2914) using the luciferase reporter construct resulted in a significant $(P<0.001)$ increase in promoter activity, which was $>18$-fold greater than the activity observed with the pGL3-Basic vector (Figure $2 a$ ). In contrast, a larger $5115 \mathrm{bp}$ genomic fragment (p5115; - 5110/+5) of the BORIS 5'-regulatory region cloned into the pGL3-Basic plasmid and transfected in 293T cells did not show any promoter activity (Figure 2a).

To determine the core BORIS promoter, we performed 5 -deletion analysis to determine the minimal region required for BORIS promoter activity (Figure $2 \mathrm{~b}$ ). The promoter activity of each of the deletion constructs was assessed by measuring luciferase activities in transiently transfected $293 \mathrm{~T}$ cells. The results showed that luciferase activity from the shorter 700-bp promoter fragment $(-1108 /-425$, p700) had approximately threefold greater activity than the full-length fragment (p2914); this p700 vector contained the $\mathrm{CpG}$ and GATA-1 regions. This observation suggests the possible existence of a strong suppressor element between -2909 and $-1108 \mathrm{nt}$ of the 
a
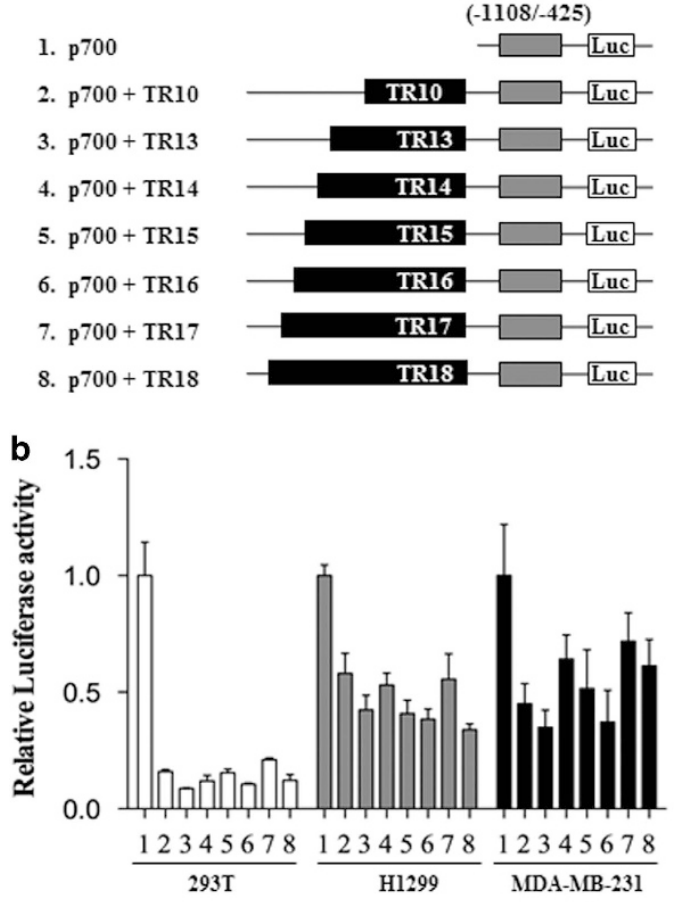

C

\begin{tabular}{c|c|c|c|c|c|c}
\hline \multirow{2}{*}{ vector } & \multicolumn{2}{|c|}{ 293T } & \multicolumn{2}{c|}{ Hl299 } & \multicolumn{2}{c}{ MDA-MB-231 } \\
\cline { 2 - 7 } & Ave. \pm SE & $P$ & Ave. \pm SE & $P$ & Ave. \pm SE & $P$ \\
\hline p 700 & $1.00 \pm 0.14$ & & $1.00 \pm 0.05$ & & $1.00 \pm 0.22$ & \\
p 700+TR10 & $0.16 \pm 0.01$ & 0.0005 & $0.58 \pm 0.09$ & $8.6 \mathrm{E}-08$ & $0.45 \pm 0.09$ & $3.42 \mathrm{E}-07$ \\
p 700+TR13 & $0.09 \pm 0.00$ & 0.0003 & $0.42 \pm 0.06$ & $4.3 \mathrm{E}-13$ & $0.35 \pm 0.07$ & $1.88 \mathrm{E}-09$ \\
p 700+TR14 & $0.12 \pm 0.02$ & 0.0004 & $0.53 \pm 0.05$ & $9.8 \mathrm{E}-12$ & $0.64 \pm 0.10$ & 0.0001 \\
p 700+TR15 & $0.15 \pm 0.02$ & 0.0005 & $0.41 \pm 0.06$ & $4.5 \mathrm{E}-13$ & $0.52 \pm 0.17$ & $2.44 \mathrm{E}-05$ \\
p 700+TR16 & $0.11 \pm 0.00$ & 0.0004 & $0.38 \pm 0.04$ & $6.4 \mathrm{E}-15$ & $0.37 \pm 0.14$ & $3.88 \mathrm{E}-07$ \\
p 700+TR17 & $0.21 \pm 0.01$ & 0.0006 & $0.56 \pm 0.11$ & $2.6 \mathrm{E}-09$ & $0.72 \pm 0.12$ & 0.4289 \\
p 700+TR18 & $0.12 \pm 0.02$ & 0.0004 & $0.34 \pm 0.02$ & $6.5 \mathrm{E}-15$ & $0.61 \pm 0.11$ & $6.21 \mathrm{E}-05$ \\
\hline
\end{tabular}

Figure 4 Effect of allelic types of BORIS-MS2 in BORIS promoter luciferase constructs. (a) The structures of the p700 (\#1) and TR (BORIS-MS2) reporter constructs. The gray square indicates the $B O R / S$ p700 promoter region, and the open square represents the open reading frame of luciferase. The black squares include the BORIS-MS2 polymorphic regions; seven different sizes of BORISMS2 (10-18 copies) were inserted in the p700 plasmid. (b) The effects of BORIS-MS2 on gene expression in the luciferase reporter system. Three different cell lines (293T, H1299 and MDA-MB-231) were transfected with eight different vectors. (c) The table shows the relative luciferase activity compared with the p700 vector as the mean \pm s.e. and the $P$-values of four independent experiments.

BORIS 5 -regulatory region, that is, the minisatellite region (Figure 2b).

A previous study ${ }^{11}$ reported three transcription start sites in the BORIS sequence-1447, 899 and $658 \mathrm{bp}$ upstream of the first ATG codon. This observation suggests the presence of three alternative promoter regions that may have cell typespecific activity. We constructed three expression cassettes (PrA, PrB and PrC) containing the predicted promoters ${ }^{11}$ and compared levels of luciferase expression in HEC293 cells (Supplementary Figure S2). The p700 vector containing the
CpG and GATA-1 regions showed the highest promoter activity, which was over 60 -fold greater than the activity observed with the pGL3-Basic vector (Supplementary Figure S2).

To obtain information on putative regulatory sites in the p700 promoter region of the BORIS gene, we analyzed the DNA fragment $(-1108 /-425)$. As shown in Figure 2, a deletion of $23 \mathrm{bp}$ from the $3^{\prime}$-end of the p700 vector (vector p678) resulted in a dramatic reduction in promoter activity. The deleted region $(-447 /-425$ region, Figure 2) includes the GATA-1-binding site and the CCAAT box. ${ }^{11}$ To assess the involvement of these putative regulatory sites in regulating the promoter activity of the BORIS gene, we introduced specific mutations in the core sequences of these sites and examined the effect of these mutations on promoter activity (Figure $3 \mathrm{a}$ ). The results show that mutating these elements led to a significant $(P<0.01)$ reduction in promoter activity compared with the p700 promoter (Figure $3 \mathrm{~b}$ ). These results suggest that the region including the GATA-1 site and the CCAAT box has a role in activating BORIS expression.

Effect of minisatellites (BORIS-MS2) on BORIS expression The comparative promoter activities of p2914 and p700 in Figure $2 \mathrm{~b}$ suggests the presence of a suppressor element located in the -2909 to -1108 region; this region corresponds to the minisatellites of BORIS-MS2. Therefore, we constructed seven different vectors (Figures 4a; p700+TRs) consisting of the p700 fragment and the BORIS-MS2 minisatellites (TR10, TR13, TR14, TR15, TR16, TR17 and TR18), which were genotyped using genomic DNA from lung cancer cases (Figure 5). For all seven constructs harboring minisatellites, the luciferase activities were significantly reduced in $293 \mathrm{~T}$ cells compared with the p700 reporter vector (5- to 10-fold reductions) (Figure 4b). Notably, when the same BORIS-MS2-containing constructs were transfected into lung cancer cells (H1299) or breast cancer cells (MDA-MB-231), we observed only a approximately twofold reduction in promoter activity (Figures $4 \mathrm{~b}$ and $\mathrm{c}$ ). This result suggests that the constructs containing the minisatellites showed different promoter activity in normal and cancer cell lines. In addition, we also constructed seven variants of the p2298 vector, which included the p700 promoter region, the region between the p700 promoter and BORIS-MS2, and different fragments of BORIS-MS2 (10-18 copies of a 56 bp repeat) (Supplementary Figure S3). These constructs were also transfected into three different cell lines, and similar results were observed. Therefore, BORIS-MS2 minisatellites may have a role in aberrant activation of BORIS during carcinogenesis. Next, we investigated a possible link between the minisatellite variants and lung cancer in a Korean population.

\section{Analysis of minisatellite variants in lung cancer patients}

Several lung cell lines and many lung tumor specimens express high levels of BORIS. ${ }^{6,8}$ Because BORIS is a potential new marker for the detection of lung cancer, we investigated whether BORIS-MS2 may correlate with lung cancer development. In a previous study, BORIS-MS2 was found to possess 
a

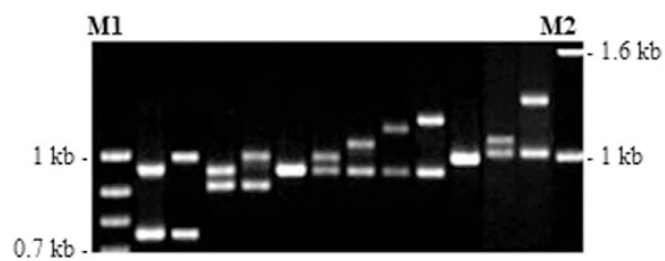

b

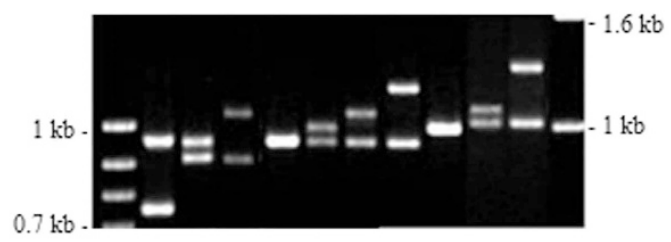

C

\begin{tabular}{c|c|c|c|c}
\hline Genotype & $\begin{array}{c}\text { Controls, } \\
N(\%)\end{array}$ & $\begin{array}{c}\text { Lung cancer } \\
\text { cases, } N(\%)\end{array}$ & OR (95\% CI) & $P$ \\
\hline $10 / 14$ & $4(0.7)$ & $2(1.0)$ & $1.44(0.26-7.90)$ & 0.676 \\
$10 / 15$ & $1(0.2)$ & $0(0.0)$ & - & 0.554 \\
$13 / 14$ & $3(0.5)$ & $6(2.9)$ & $5.87(1.45-23.69)$ & $0.005 *$ \\
$13 / 15$ & $3(0.5)$ & $0(0.0)$ & - & 0.305 \\
$13 / 16$ & $0(0.0)$ & $1(0.5)$ & - & 0.09 \\
$14 / 14$ & $232(39.3)$ & $86(41.7)$ & $1.11(0.80-1.53)$ & 0.541 \\
$14 / 15$ & $246(41.7)$ & $84(40.8)$ & $0.96(0.70-1.33)$ & 0.818 \\
$14 / 16$ & $2(0.3)$ & $1(0.5)$ & $1.43(0.13-15.90)$ & 0.768 \\
$14 / 17$ & $1(0.2)$ & $0(0.0)$ & - & 0.554 \\
$14 / 18$ & $3(0.5)$ & $1(0.5)$ & $0.95(0.10-9.23)$ & 0.968 \\
$15 / 15$ & $92(15.6)$ & $23(11.2)$ & $0.68(0.42-1.11)$ & 0.12 \\
$15 / 16$ & $1(0.2)$ & $1(0.5)$ & $2.87(0.18-46.15)$ & 0.435 \\
$15 / 18$ & $2(0.3)$ & $1(0.5)$ & $1.43(0.13-15.90)$ & 0.768 \\
\hline
\end{tabular}

Figure 5 Haplotyping of BORIS-MS2 in cancer-free controls and lung cancer patients. (a) Electrophoretic patterns of PCR products of BORIS-MS2 in controls. Seven BORIS-MS2 alleles and 12 haplotype patterns were detected in DNA from 590 cancer-free female controls. (b) Electrophoretic patterns of PCR products of BORIS-MS2 in lung cancer patients. Six BORIS-MS2 alleles and 10 haplotype patterns were detected in the DNA from 206 patients with lung cancer. The first and last lanes correspond to a 100-bp (M1; Invitrogen) and a 1-kb size marker (M2; Invitrogen). (c) Allelic genotypes and frequency in controls and cases with lung cancer. Bold numbers represent the rare alleles of BORIS-MS2. ${ }^{*} P<0.05$.

genetically variable alleles. ${ }^{6,12}$ In this study, we investigated whether these alleles are linked to activation of BORIS during tumorigenesis. For this purpose, the frequency of polymorphic BORIS-MS2 alleles was compared between controls and patients with lung cancer in a Korean population (Table 1). This analysis included PCR amplification of the minisatellite from human genomic DNA samples isolated from 590 cancerfree controls and 206 patients with lung cancer (Table 1). BORIS-MS2 had 12 types of haploid patterns in controls (Figure 5a) and 10 types of haploid patterns in cancer cases (Figure $5 b$ ). Figure $5 c$ summarizes the frequency of haploid patterns for BORIS-MS2 between the controls and the lung cancer groups. Among the identified haploid patterns, the 13/14 genotype of BORIS-MS2 (corresponding to 13 copies of the satellite repeat on 1 chromosome and 14 copies of the satellite repeat on another chromosome) was significantly more frequent in cases $(2.9 \%)$ than in controls $(0.5 \%)(P<0.005)$, and for the frequency of the allelic distribution (Table 2) 13 repeated alleles of BORIS-MS2 were significantly more frequent in cases than in controls ( 0.5 vs $1.7 \%)$. Analysis of these data revealed a significant association between 13 tandem repeat alleles and the odds for lung cancer (BORIS-MS2 and lung cancer OR: 3.38, CI: 1.13-10.12; $P=0.021$ ).

For further analysis, each BORIS-MS2 allele was divided into common or rare alleles (the frequency for rare alleles was $<1 \%$ ) based on their frequency in the control population. ${ }^{12}$ Seven alleles of BORIS-MS2 were grouped into 2 common alleles (14 and 15 repeats) and 5 rare alleles (10, 13 16, 17 and 18 repeats) in this study (Table 3). There was a significant difference in the frequency of rare alleles found in controls and cancer cases (OR: 2.04, CI: 1.02-4.08; $P=0.039$ ). Specifically, the frequency of rare alleles in female cases with lung cancer showed a statistically significant difference between controls and cases $(\mathrm{OR}=3.14 ; P=0.047)$. Furthermore, we divided the rare alleles into short (10 and 13) and long (16, 17 and 18) groups based on their tandem repeat lengths (Table 4). The analysis of the short rare alleles (10 and 13 repeats) group, comparing controls and cases, also showed an association, with a relative lung cancer OR of $2.37(P=0.049)$. There was a more significant difference in the frequency of short rare alleles between male controls and male cases $(\mathrm{OR}=3.86 ; P=0.041)$ (Table 4).

We used clinicopathological information obtained in 2009 from the Korea University Anam Hospital in Korea ${ }^{19}$ and between 2002 and 2007 from Dong-A University and Chungbuk National University Hospital in Korea (Supplementary Table S2). Tumors, nodes and distant metastasis stages were analyzed according to the World Health Organization (WHO) system. Lung tumors were categorized into the appropriate class, and we then assessed the frequency of each class in the entire cancer group and the rare allele group. There was no association between short rare alleles and cancer classification corresponding to stage, $\mathrm{T}$ stage, $\mathrm{N}$ stage, $\mathrm{M}$ stage or cell type.

\section{DISCUSSION}

BORIS is a CTA gene family member, and its transcription is abnormally activated in various tumors and cancer cells. ${ }^{2,4-6,20,21}$ It was also reported that the expression of BORIS is predominantly controlled by DNA methylation ${ }^{1}$ and that BORIS mediates the epigenetic regulation of other genes (NY-ESO-1, NOTCH3, hTERT and so on) in cancer cells. ${ }^{8,22,23}$ In our previous study, however, no correlation between methylation status and BORIS gene expression was detected in tissues from gastric cancer. ${ }^{6}$ Similar data were obtained in this work based on analysis of tissues from lung cancer. Based on these results, we suggest that the expression of BORIS is regulated by more complex epigenetic mechanisms.

To elucidate the regulation of BORIS beyond DNA methylation, we physically characterized the entire BORIS locus. A CpG island ( -1096 to -762 from the first ATG codon) and two minisatellites (variable number of tandem repeats; BORIS-MS1 and BORIS-MS2) were identified through the 
Table 2 Comparison of allelic sizes and frequency of Boris-MS2

\begin{tabular}{|c|c|c|c|c|c|c|c|c|c|}
\hline \multirow[b]{2}{*}{$T R$} & \multirow[b]{2}{*}{ Size $(b p)$} & \multicolumn{3}{|c|}{ Controls, N (frequency) } & \multicolumn{3}{|c|}{ Lung cancer cases, N (frequency) } & \multirow[b]{2}{*}{ OR $(95 \% \mathrm{Cl})$} & \multirow[b]{2}{*}{ P-value } \\
\hline & & $\begin{array}{c}\text { Male } \\
\mathrm{N}=652\end{array}$ & $\begin{array}{c}\text { Female } \\
\mathrm{N}=528\end{array}$ & $\begin{array}{c}\text { Total } \\
\mathrm{N}=1180\end{array}$ & $\begin{array}{c}\text { Male } \\
\mathrm{N}=342\end{array}$ & $\begin{array}{l}\text { Female } \\
\mathrm{N}=70\end{array}$ & $\begin{array}{c}\text { Total } \\
\mathrm{N}=412\end{array}$ & & \\
\hline 10 & 785 & $1(0.002)$ & $4(0.008)$ & $5(0.004)$ & $1(0.003)$ & $1(0.014)$ & $2(0.005)$ & $1.15(0.22-5.93)$ & 0.871 \\
\hline 13 & 935 & $2(0.003)$ & $4(0.008)$ & $6(0.005)$ & $5(0.015)$ & $2(0.029)$ & $7(0.017)$ & $3.38(1.13-10.12)$ & $0.021 *$ \\
\hline 16 & 1100 & $2(0.003)$ & $1(0.002)$ & $3(0.003)$ & $3(0.009)$ & - & $3(0.007)$ & $2.88(0.58-14.31)$ & 0.177 \\
\hline 17 & 1160 & $1(0.002)$ & - & $1(0.001)$ & - & - & - & - & 0.554 \\
\hline 18 & 1220 & $4(0.006)$ & $1(0.002)$ & $5(0.004)$ & $1(0.003)$ & $1(0.014)$ & $2(0.005)$ & $1.15(0.22-5.93)$ & 0.871 \\
\hline
\end{tabular}

Abbreviations: $\mathrm{Cl}$, confidence interval; OR, odds ratio; TR, tandem repeat. $* P<0.05$.

Table 3 The frequency of rare Boris-MS2 alleles in controls and lung cancer cases

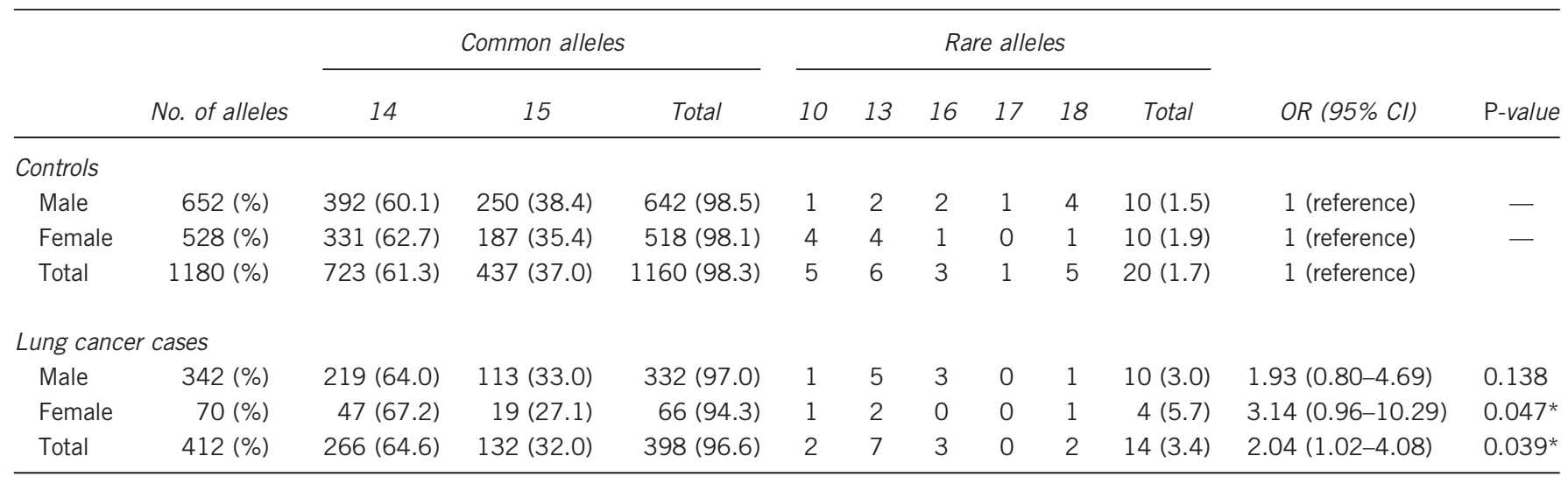

Abbreviations: $\mathrm{Cl}$, confidence interval; $\mathrm{OR}$, odds ratio.

${ }^{*} P<0.05$.

Table 4 The frequency of short rare Boris-MS2 alleles in controls and lung cancer cases

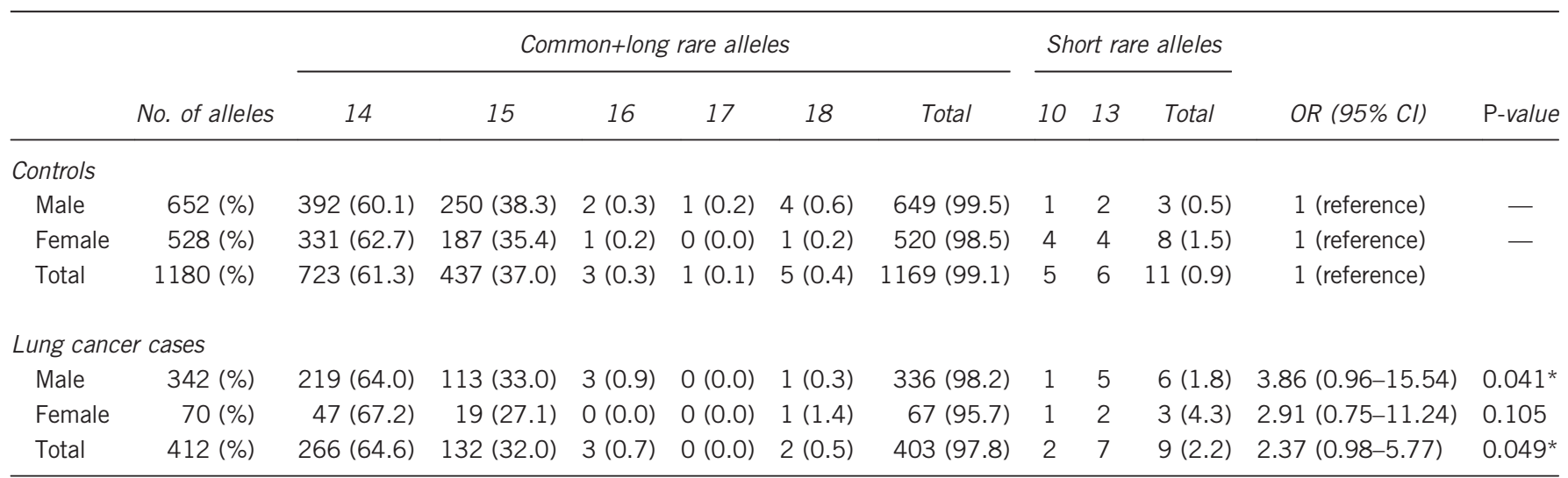

Abbreviations: $\mathrm{Cl}$, confidence interval; $\mathrm{OR}$, odds ratio.

${ }^{*} P<0.05$.

characterization of the genomic DNA sequence upstream of the gene. $^{12}$ In this study, we examined the $\sim 5.5-\mathrm{kb}$ region upstream of the start codon of BORIS. A set of luciferase reporter cassettes carrying fragments from the upstream region of various sizes were constructed and tested (Figure 2). The highest luciferase activity was detected with a 700-bp promoter fragment $(-1108 /-425$, p700) which contained CpG islands, a GATA-1 site and CCAAT box regions. This finding is in 
agreement with a previous study, ${ }^{11}$ where three transcription start sites were mapped within the same 5'-upstream region. However, our analysis revealed a potential suppressor between -2909 and $-1108 \mathrm{nt}$ of the BORIS $5^{\prime}$-regulatory region containing the minisatellite region of BORIS-MS2 (Figure $2 \mathrm{~b}$ ). This finding suggests that the transcriptional regulation of BORIS is more complicated and that other types of regulation may exist in addition to DNA methylation. Moreover, there are also several reports indicating that minisatellite polymorphisms can influence gene expression. ${ }^{16,17,24-28}$

In separate experiments, we investigated how polymorphic variants of BORIS-MS2 minisatellite affect the transcriptional activity of BORIS in cell lines using a luciferase reporter system (Figure 4). In 293T normal cell lines, all seven minisatellite constructs (TR10, 13,14,15,16, 17 and 18) significantly decreased (5-10 times reduction) the activity of the p700 BORIS promoter, but there was no clear relationship between the tandem repeat copy number and level of gene expression. In principle, this result may be suggestive of the restriction of in vivo experiments. However, when the same constructs were transfected in lung cancer cells (H1299) or breast cancer cells (MDA-MB-231), the promoter activity was reduced only approximately two times compared with the control. This result suggests that the expression cassettes carrying BORIS-MS2 are controlled in a different way in normal and cancer cell lines. Similar results for transcriptional regulation by minisatellite fragments were previously reported with $H$-Ras, IL-1 $\alpha$, IGF2, MUC6 and TERT genes. ${ }^{16,17,24-26}$ Minisatellites of IL-1 $\alpha$, MUC6 and TERT may control gene expression in cancer cell lines, although these are located in the intronic regions of genes. ${ }^{16,17}$ We suggest that BORIS-MS2, located in the promoter region, differentially regulates the gene transcription in cancer cells and normal cells. This suggests a potential association between BORIS-MS2 and cancer. For example, specific alleles of BORIS-MS2 may affect susceptibility to lung cancer. In this study, we found a significantly elevated frequency of rare alleles of the minisatellite in lung cancer cases compared with cancer-free controls. In addition, short rare minisatellite alleles were associated with higher susceptibility in lung cancer patients. A similar result has been shown in a report examining the relationship between short rare alleles of BORIS-MS2 and breast cancer in a younger group. ${ }^{12}$ Other studies on genetic susceptibility due to specific minisatellites have also been reported. ${ }^{17,29-31}$

It is commonly accepted that genomic instability has a crucial role in the accumulation of genetic alterations that are responsible for cancer cell development. ${ }^{32}$ It has also been reported that genomic instability in minisatellite regions is related to loss of heterozygosity and rearrangements in cancer tissues. $^{33,34}$ Thus, the BORIS-MS2 minisatellites may be involved in tumorigenesis not only via gene regulation but also via genomic instability. Further analysis is required to elucidate the correlation between rare minisatellite alleles and the susceptibility to lung cancer observed in this study.

\section{CONFLICT OF INTEREST}

The authors declare no conflict of interest.

\section{ACKNOWLEDGEMENTS}

We gratefully acknowledge the patients, families and their caregivers for their willing participation in this project and for providing consent for the use of their information in this study. The study design and procedures were approved by the Committee of Bioethics at Dong-A University (\#IRB-06-10-02 \& IRB-07-10-7; Busan, Korea). This research was supported by the Mid-Career Researcher Program through the National Research Foundation of Korea (NRF) grant (NRF-2013R1A2A2A04008115) funded by the Korea government (MEST). This work was supported by the Brain Busan 21 Project in 2015. SIK was supported by the Bio-Synergy Research Project (NRF- 2014M3A9C4066461) of the Ministry of Science, ICT and Future Planning through the National Research Foundation and the Korea Basic Science Institute research program (D35402).

1 Loukinov DI, Pugacheva E, Vatolin S, Pack SD, Moon H, Chernukhin I et al. BORIS, a novel male germ-line-specific protein associated with epigenetic reprogramming events, shares the same 11 -zinc-finger domain with CTCF, the insulator protein involved in reading imprinting marks in the soma. Proc Natl Acad Sci USA 2002; 99: 6806-6811.

2 Simpson AJ, Caballero OL, Jungbluth A, Chen YT, Old LJ. Cancer/testis antigens, gametogenesis and cancer. Nat Rev Cancer 2005; 5: 615-625.

3 D'Arcy V, Pore N, Docquier F, Abdullaev ZK, Chernukhin I, Kita GX et al. BORIS, a paralogue of the transcription factor, CTCF, is aberrantly expressed in breast tumours. Br J Cancer 2008; 98: 571-579.

4 Vatolin S, Abdullaev Z, Pack SD, Flanagan PT, Custer M, Loukinov DI et al. Conditional expression of the CTCF-paralogous transcriptional factor BORIS in normal cells results in demethylation and derepression of MAGE-A1 and reactivation of other cancer-testis genes. Cancer Res 2005; 65: 7751-7762.

5 Risinger JI, Chandramouli GV, Maxwell GL, Custer M, Pack S, Loukinov D et al. Global expression analysis of cancer/testis genes in uterine cancers reveals a high incidence of BORIS expression. Clin Cancer Res 2007; 13: 1713-1719.

6 Yoon SL, Roh YG, Lee SH, Kim SH, Kim MC, Kim SJ et al. Analysis of promoter methylation and polymorphic minisatellites of BORIS and lack of association with gastric cancer. DNA Cell Biol 2011; 30: 691-698.

7 D'Arcy V, Abdullaev ZK, Pore N, Docquier F, Torrano V, Chernukhin I et al. The potential of BORIS detected in the leukocytes of breast cancer patients as an early marker of tumorigenesis. Clin Cancer Res 2006; 12: 5978-5986.

8 Hong JA, Kang Y, Abdullaev Z, Flanagan PT, Pack SD, Fischette MR et al. Reciprocal binding of CTCF and BORIS to the NY-ESO-1 promoter coincides with derepression of this cancer-testis gene in lung cancer cells. Cancer Res 2005; 65: 7763-7774.

9 Hoffmann MJ, Muller M, Engers R, Schulz WA. Epigenetic control of CTCFL/BORIS and OCT4 expression in urogenital malignancies. Biochem Pharmacol 2006; 72: 1577-1588.

10 Woloszynska-Read A, James SR, Link PA, Yu J, Odunsi K, Karpf AR. DNA methylation-dependent regulation of BORIS/CTCFL expression in ovarian cancer. Cancer Immun 2007; 7: 21.

11 Renaud S, Pugacheva EM, Delgado MD, Braunschweig R, Abdullaev Z, Loukinov D et al. Expression of the CTCF-paralogous cancer-testis gene, brother of the regulator of imprinted sites (BORIS), is regulated by three alternative promoters modulated by CpG methylation and by CTCF and p53 transcription factors. Nucleic Acids Res 2007; 35: 7372-7388.

12 Yoon SL, Kim DC, Cho SH, Lee SY, Chu IS, Heo J et al. Susceptibility for breast cancer in young patients with short rare minisatellite alleles of BORIS. BMB Rep 2010; 43: 698-703.

13 Bresnick EH, Katsumura KR, Lee HY, Johnson KD, Perkins AS. Master regulatory GATA transcription factors: mechanistic principles and emerging links to hematologic malignancies. Nucleic Acids Res 2012; 40: 5819-5831. 
14 Dolfini D, Mantovani R. Targeting the Y/CCAAT box in cancer: YB-1 (YBX1) or NF-Y? Cell Death Differ 2013; 20: 676-685.

15 Jemal A, Bray F, Center MM, Ferlay J, Ward E, Forman D. Global cancer statistics. CA Cancer J Clin 2011; 61: 69-90.

16 Kwon JA, Lee SY, Ahn EK, Seol SY, Kim MC, Kim SJ et al. Short rare MUC6 minisatellites- 5 alleles influence susceptibility to gastric carcinoma by regulating gene. Hum Mutat 2010; 31: 942-949.

17 Yoon SL, Jung SI, Do EJ, Lee SR, Lee SY, Chu IS et al. Short rare hTERT-VNTR2-2nd alleles are associated with prostate cancer susceptibility and influence gene expression. BMC Cancer 2010; 10: 393.

18 Kouprina N, Larionov V. Selective isolation of genomic loci from complex genomes by transformation-associated recombination cloning in the yeast Saccharomyces cerevisiae. Nat Protoc 2008; 3: 371-377.

19 Sung JS, Whang YM, Park KH, Ryu JS, Choi JG, Seo JH et al. No association between promoter polymorphism of STK11 gene and lung cancer risk in the Korean population. Cancer Res Treat 2009; 41: 211-217.

20 Cheema Z, Hari-Gupta Y, Kita GX, Farrar D, Seddon I, Corr J et al. Expression of the cancer-testis antigen BORIS correlates with prostate cancer. Prostate 2014; 74: 164-176.

21 Martin-Kleiner I. BORIS in human cancers-a review. Eur J Cancer 2012; 48: 929-935.

22 Zampieri M, Ciccarone F, Palermo R, Cialfi S, Passananti C, Chiaretti S et al. The epigenetic factor BORIS/CTCFL regulates the NOTCH3 gene expression in cancer cells. Biochim Biophys Acta 2014; 1839: 813-825.

23 Renaud S, Loukinov D, Alberti L, Vostrov A, Kwon YW, Bosman FT et al. BORIS/CTCFL-mediated transcriptional regulation of the hTERT telomerase gene in testicular and ovarian tumor cells. Nucleic Acids Res 2011; 39: 862-873.

24 Trepicchio WL, Krontiris TG. Members of the rel/NF-kappa B family of transcriptional regulatory proteins bind the HRAS1 minisatellite DNA sequence. Nucleic Acids Res 1992; 20: 2427-2434.

25 Paquette J, Giannoukakis N, Polychronakos C, Vafiadis P, Deal C. The INS $5^{\prime}$ variable number of tandem repeats is associated with IGF2 expression in humans. J Biol Chem 1998; 273: 14158-14164.

26 Bailly S, Israel N, Fay M, Gougerot-Pocidalo MA, Duff GW. An intronic polymorphic repeat sequence modulates interleukin-1 alpha gene regulation. Mol Immunol 1996; 33: 999-1006.
27 Fiskerstrand CE, Lovejoy EA, Quinn JP. An intronic polymorphic domain often associated with susceptibility to affective disorders has allele dependent differential enhancer activity in embryonic stem cells. FEBS Lett 1999; 458: 171-174.

28 Nakamura Y, Koyama K, Matsushima M. VNTR (variable number of tandem repeat) sequences as transcriptional, translational, or functional regulators. J Hum Genet 1998; 43: 149-152.

29 Ahn EK, Kim WJ, Kwon JA, Choi PJ, Kim WJ, Sunwoo Y et al. Variants of MUC5B minisatellites and the susceptibility of bladder cancer. DNA Cell Biol 2009; 28: 169-176.

30 Ahn MH, Bae KB, Kwon JA, Choi HJ, Lee SR, Kim SH et al. Association of MUC6-minisatellite variants with susceptibility to rectal carcinoma. Mol Biol Rep 2013; 40: 303-308.

31 Seol SY, Lee SY, Kim YD, Do EJ, Kwon JA, Kim SI et al. Minisatellite polymorphisms of the SLC6A19: susceptibility in hypertension. Biochem Biophys Res Commun 2008; 374: 714-719.

32 Lengauer C, Kinzler KW, Vogelstein B. Genetic instabilities in human cancers. Nature 1998; 396: 643-649.

33 Jeong YH, Kim MC, Ahn EK, Seol SY, Do EJ, Choi HJ et al. Rare exonic minisatellite alleles in MUC2 influence susceptibility to gastric carcinoma. PLOS ONE 2007; 2: e1163.

34 Szutorisz H, Palmqvist R, Roos G, Stenling R, Schorderet DF, Reddel R et al. Rearrangements of minisatellites in the human telomerase reverse transcriptase gene are not correlated with its expression in colon carcinomas. Oncogene 2001; 20: 2600-2605.

(c) (1) (5) $\Theta$ This work is licensed under a Creative Commons Attribution-NonCommercial-NoDerivs 4.0 International License. The images or other third party material in this article are included in the article's Creative Commons license, unless indicated otherwise in the credit line; if the material is not included under the Creative Commons license, users will need to obtain permission from the license holder to reproduce the material. To view a copy of this license, visit http://creativecommons.org/licenses/by-nc-nd/4.0/

Supplementary Information accompanies the paper on Experimental \& Molecular Medicine website (http://www.nature.com/emm) 